\title{
COMPORTAMENTO DO CONSUMIDOR DE ALIMENTOS ORGÂNICOS: UM MODELO BASEADO EM INFLUENCIADORES DIRETOS E INDIRETOS
}

\author{
Nicoline Pinheiro Fernandes \\ Mestre em Administração \\ Universidade Federal do Rio Grande Rio Grande - RS - Brasil \\ nicolinefer@gmail.com https://orcid.org/0000-0003-1713-8244 \\ Guilherme Lerch Lunardi \\ Doutor em Administração \\ Universidade Federal do Rio Grande Rio Grande - RS - Brasil \\ gllunardi@furg.br http://orcid.org/0000-0003-3250-2796 \\ Aléxia Fernandes Rocha \\ Mestre em Administração \\ Universidade Federal do Rio Grande Rio Grande - RS - Brasil \\ rochaalexiaf@gmail.com http://orcid.org/0000-0001-6193-3785 \\ Suzi Samá \\ Doutora em Educação em Ciências \\ Universidade Federal do Rio Grande Rio Grande - RS - Brasil \\ suzisama@furg.br http://orcid.org/0000-0002-7490-9722
}

\section{RESUMO}

O crescimento da agricultura orgânica, seguido pelo aumento de consumidores interessados em tais produtos e do número de estabelecimentos que os comercializam atualmente, instigaram este estudo a analisar diferentes determinantes do comportamento do consumidor de alimentos orgânicos, pela proposição de um modelo estrutural que explique e preveja fatores que influenciam, direta e indiretamente, o consumo efetivo destes produtos. Para isso, realizou-se uma pesquisa survey em diferentes grupos e comunidades de produtos orgânicos presentes na rede social Facebook. Os dados foram analisados por meio da técnica de modelagem de equações estruturais, baseada nos mínimos quadrados parciais (PLS). Os resultados apontaram que a qualidade do produto, consciência ecológica do consumidor e conhecimento sobre produtos orgânicos, a disponibilidade do produto e o tempo que os consumidores consomem orgânicos influenciam positivamente o consumo desses alimentos, tanto em termos de variedade de produtos como de valores gastos. Identificou-se, ainda, um efeito moderador do preço na relação entre a atitude dos consumidores frente aos alimentos orgânicos e o seu comportamento de compra, sugerindo que quanto melhor for o preço desses produtos para o consumidor, cuja atitude em relação a eles seja favorável, maior será o seu consumo.

Palavras-chave: Alimentos orgânicos. Comportamento do consumidor. Modelagem de equações estruturais. Produtos orgânicos.

\section{CONSUMER BEHAVIOR OF ORGANIC FOODS: A MODEL BASED ON DIRECT AND INDIRECT INFLUENCERS}

\begin{abstract}
The growth of organic agriculture, followed by the increase of consumers interested in such products and the number of commercial establishments that currently sell them, instigated us to analyze different determinants of the consumer behavior of organic foods, by proposing a structural model that explains and predict factors that directly and indirectly influence the actual consumption of these products. For this, we surveyed different members of groups and communities interested in organic products present in the social network Facebook. The data were analyzed using the structural equation modeling technique, based on partial least squares (PLS). We found that consumers' attitude toward organic products (been influenced by the quality of the product, ecological awareness of the consumer, and knowledge about organics), product availability, and time this kind of food is consumed influence positively the consumption of organic food both in terms of product variety and amount spent. We also identified a moderating effect of price in the relationship between consumers' attitude and their buying behavior, suggesting that the better the price of these products for consumers whose attitude toward organics is favorable, the greater their consumption.
\end{abstract}

Keywords: Consumer behavior. Organic food. Organic products. Structural equation modeling.

Data da submissão: $18 / 02 / 2020$

Data de aceite: 04/06/2020

Revista de Gestão Social e Ambiental - RGSA, São Paulo, v. 14, n. 2, p. 48-64, maio/ago. 2020. 


\section{INTRODUÇÃO}

O consumo de alimentos tem sofrido alterações, de acordo com as inovações agroindustriais e as características sociais, culturais e econômicas da população, bem como o crescimento da preocupação dos consumidores com aspectos ligados à saúde, bem-estar e sustentabilidade (Olson, 2017; Pereira, Lima-Filho, Maciel, \& Oliveira, 2015). Nessa perspectiva, encontram-se os alimentos orgânicos, que são produzidos sem a utilização de agrotóxicos ou outro produto químico, podendo ser de origem vegetal ou animal, e que, ao invés do uso de agentes químicos, utilizam práticas alternativas de cultura, as quais proporcionam equilíbrio no sistema agrícola, sob o ponto de vista ecológico (Mapa, 2015).

No Brasil, o cultivo de alimentos orgânicos vem ganhando cada vez mais espaço, crescendo a uma taxa média de $15 \%$ ao ano, o qual movimentou, em 2019, R\$ 4,5 bilhões (Soares \& Marzzaro, 2020). Segundo o Ministério da Agricultura, o número de produtores orgânicos registrados no Brasil já chega a 27,9 mil, dos quais cerca de $70 \%$ deles vem da agricultura familiar (Boehm, 2018; Soares \& Marzzaro, 2020). Os consumidores têm sido considerados os principais impulsionadores desse movimento (Rana \& Paul, 2017), seja pela maneira como os alimentos orgânicos são produzidos, podendo ser considerada uma forma alternativa àquela chamada convencional (buscando a preservação do meio ambiente, a sustentabilidade e a manutenção de um sistema que garanta a sobrevivência das próximas gerações), seja pelo caráter individual, pois questões relacionadas à saúde de cada indivíduo fazem com que o consumidor - ainda que ocasional - opte pelo alimento orgânico, não necessariamente por questões sociais, ecológicas ou coletivas, mas por oferecerem menor risco à saúde ou, ainda, por perceberem este tipo de alimento como mais saboroso (Olson, 2017; Rana \& Paul, 2017).

Frequentemente, os estudos relacionados aos produtos orgânicos abordam a influência de diferentes elementos na intenção ou no comportamento de compra e consumo de seus consumidores (Bashaa, Mason, Shamsudin, Hussain, \& Salem, 2015; Paul \& Rana, 2012; Sampaio \& Gosling, 2014). Algumas dessas pesquisas apontam a preocupação com a saúde (Figueiró, Batistella, Silva, Saldanha, \& Slongo, 2012; Rana \& Paul, 2017), com o meio ambiente (Barcelos, Bossle, Perin, \& Vieira, 2015; Bashaa et al., 2015; Dias, Schultz, Schuster, Talamini, \& Révillion, 2015) e com a qualidade dos alimentos (Bashaa et al., 2015; Zamberlan, Büttenbender, Sparemberger, \& Damer, 2010) como importantes fatores que influenciam o consumo desses produtos. A literatura sugere, ainda, que outros elementos, tais como o conhecimento sobre as características e os benefícios dos alimentos orgânicos (Aschemann-Witzel \& Zielke, 2017), o preço (Oluwoye, Chembezi, \& Herbert, 2017; Sampaio \& Gosling, 2014) e a disponibilidade do produto (Dettmann \& Dimitri, 2010; Hoppe, Vieira, \& Barcellos, 2013; Zamberlan et al., 2010) também influenciam as pessoas a consumir esse tipo de alimento.

De forma complementar, Hoppe, Barcellos, Vieira e Matos (2012) e Rana e Paul (2017) evidenciaram em suas pesquisas que a atitude favorável aos alimentos orgânicos é um dos principais preditores da intenção de compra desses produtos, ainda que considerem que muitos indivíduos possuem atitudes positivas sobre estes alimentos, mas nem sempre os compram, sugerindo a existência de outros fatores ou características que influenciam a sua decisão final. Peattie (2015) descreve esse problema entre a inconsistência sobre o que as pessoas afirmam (ou expressam, através de valores, crenças e atitudes) e o que realmente fazem como uma lacuna entre o comportamento do consumidor e a sua intenção de compra frente aos alimentos orgânicos. Com isso, muitos pesquisadores têm destacado a necessidade de pesquisas mais aprofundadas, de modo a enfrentar esse desafio e diminuir as lacunas existentes entre a atitude e o comportamento real dos consumidores - especialmente, reavaliando potenciais fatores que influenciam o consumo real e não apenas a intenção de compra desses alimentos (Chekima, Chekima \& Chekima, 2019). 
Nesse sentido, este artigo contribui com a literatura ao analisar diferentes determinantes do comportamento do consumidor de produtos orgânicos, por meio da proposição de um modelo estrutural que explique e preveja fatores que influenciam, direta e indiretamente, o consumo efetivo destes produtos. $\mathrm{O}$ estudo se justifica pelo aumento do consumo de alimentos orgânicos, tanto no Brasil como no mundo, e pelo interesse das pessoas nesse tipo de cultura. Observa-se que a sua disponibilidade cresce a cada ano, nos mais diversos locais, sendo oferecido em lancherias, cafés e restaurantes voltados especificamente a este nicho de mercado, destacando-se, ainda, o aumento de feiras permanentes ou itinerantes, e até mesmo grandes redes de supermercados, que disponibilizam produtos orgânicos diretamente aos consumidores. Portanto, entender os fatores que influenciam o consumo real dos indivíduos que adquirem e consomem alimentos orgânicos pode ser uma boa alternativa para ampliar o conhecimento sobre o comportamento ambiental desses consumidores, sendo útil a produtores, comerciantes e legisladores em seus esforços para promover o consumo de alimentos orgânicos entre os cidadãos.

\section{FUNDAMENTAÇÃO TEÓRICA}

Nesta seção, são discutidos aspectos relacionados ao comportamento do consumidor de produtos orgânicos, seguido das hipóteses propostas e o modelo conceitual da pesquisa.

\subsection{Comportamento do consumidor de produtos orgânicos}

A partir da década de 1960, motivada por uma constante preocupação sustentável e ambiental da sociedade, e somada a uma série de barreiras sanitárias impostas no mundo, houve um aumento da demanda por parte dos consumidores por serviços e produtos que proporcionem melhor bem-estar e saúde, ocasionando, assim, uma expansão do mercado mundial de produtos naturais e orgânicos (Dias et al., 2015). Pato e Tamayo (2006) apontam que esse comportamento ecológico possui fatores multidimensionais, predominando aspectos ligados ao consumo, ativismo, economia de recursos, tais como água e energia, limpeza urbana e reciclagem.

O comportamento do consumidor de alimentos, por sua vez, também é influenciado por este contexto, estando relacionado diretamente ao seu ambiente, a sua família e cultura. Com base em estudos prévios, observa-se que os alimentos orgânicos simbolizam mais do que somente a sua função fisiológica, representando também uma preocupação com a saúde do indivíduo e sua família, com a segurança dos alimentos, sabor e cuidado com o meio ambiente (Hoppe et al., 2012). Tendo estes conceitos em mente, este trabalho concentra-se, principalmente, nas influências que os consumidores sofrem de vários meios e que, por sua vez, acabam interferindo no seu processo de compra e consumo. Torna-se interessante, nesse sentido, entender o que é o mercado de produtos orgânicos, quem são os seus consumidores e quais fatores influenciam a sua tomada de decisão.

Segundo Cardoso e Rodrigues (2015), produtos orgânicos são alimentos que apresentam maior qualidade aliada à alta taxa nutricional e sem o uso de agrotóxicos, ou qualquer produto que possa causar contaminação do solo e dos alimentos. Geralmente, os consumidores de tais produtos são indivíduos conscientes e interessados em questões ecológicas, que acreditam que todos os produtos e serviços impactam o meio ambiente e, com isso, alteram seu comportamento de compra e consumo para uma forma mais ecológica, tentando diminuir o dano ambiental o máximo possível (Wee, Ismail, \& Ishak, 2014). Percebe-se que a produção de orgânicos está alinhada a uma sustentabilidade ambiental e social, atrelando aos benefícios ambientais e de saúde, a viabilidade econômica e o desenvolvimento da agricultura familiar.

Sampaio e Gosling (2014) destacam que os produtos orgânicos, como os hortifrutis, por exemplo, apresentam pouca influência da marca, não dependendo de uma logomarca para serem comercializados. Quando comparados aos produtos convencionais, o consumidor entende que se trata de produtos saudáveis e seguros (Rana \& Paul, 2017), remetendo a ligação dos orgânicos à ideia de preservação dos recursos naturais e desenvolvimento local, além de serem alimentos mais

Revista de Gestão Social e Ambiental - RGSA, São Paulo, v. 14, n. 2, p. 48-64, maio/ago. 2020. 
saudáveis e saborosos (Dias et al., 2015; Olson, 2017; Thøgersen, Barcellos, Perin, \& Zhou, 2015). Quanto ao preço desses produtos, alguns estudos têm mostrado que os consumidores até estão dispostos a arcar com um valor maior para a sua aquisição, desde que suas expectativas sejam atendidas (Aschemann-Witzel \& Zielke, 2017; Oluwoye et al., 2017; Rana \& Paul, 2017). Ainda assim, este fator representa uma importante barreira para a compra de alimentos orgânicos (Thøgersen, Pedersen, Paternoga, Schwendel, \& Aschemann-witzel, 2017), afastando, por vezes, consumidores ou até mesmo potenciais consumidores. Já as pesquisas de Hoppe et al. (2012), Hoppe et al. (2013) e Oluwoye et al. (2017) destacam a influência de alguns fatores, tais como a disponibilidade do produto orgânico e o conhecimento do consumidor quanto às características e benefícios de tais produtos, como importantes aspectos que influenciam o seu consumo. Segundo Rana e Paul (2017), vários destes fatores seriam capazes de modificar a atitude dos consumidores em relação aos produtos orgânicos e ao seu consumo. Assim, a partir de uma extensa revisão da literatura nacional e internacional sobre o tema, foram levantados potenciais influenciadores diretos e indiretos do consumo de alimentos orgânicos, os quais foram testados empiricamente nesta pesquisa. A seguir, apresenta-se o desenvolvimento das hipóteses que sustentam o modelo conceitual proposto.

\subsection{Fatores influenciadores do consumo de alimentos orgânicos}

De acordo com Engel, Blackwell e Miniard (2000), é essencial compreender o que os consumidores gostam e não gostam em um determinado produto. Este aspecto é denominado atitude, podendo ser definida como uma avaliação geral do produto. Hoppe et al. (2013) explicam que a atitude pode ser entendida pelo afeto, ou seja, pelas emoções e pelos sentimentos que o consumidor tem pelo produto; que se relaciona com os aspectos cognitivos, isto é, o conhecimento, as crenças e opiniões a respeito desse bem; a qual está atrelada à ação comportamental. Assim, ao combinar esses três conceitos, o consumidor apresenta uma determinada atitude, podendo ser ela favorável ou não, dependendo de seus sentimentos e crenças sobre os atributos do produto.

A atitude pode influenciar o comportamento de compra, por meio de elementos abstratos (crenças, sentimentos) e de ações concretas que construirão a intenção de consumir ou não determinado produto, e esta, por sua vez, determinará o ato de adquirir o item (Bagozzi, 1981). Portanto, tanto atitude quanto intenção comportamental podem explicar, direta ou indiretamente, o comportamento da compra de alimentos orgânicos. Rana e Paul (2017) destacam certos trabalhos em que a atitude aparece como o principal preditor da intenção de compra de alimentos orgânicos, mesmo que tenham evidenciado que muitas pessoas possuem atitudes positivas sobre estes alimentos, mas nem sempre os compram, evidenciando certa incoerência entre atitude e comportamento. Ainda assim, os valores de consumo influenciam as atitudes e percepções dos consumidores quanto aos alimentos orgânicos (Nasir \& Karakaya, 2014), havendo fatores críticos que contribuem para a formação dessas atitudes e comportamentos, como a qualidade percebida do produto, a preocupação com o meio ambiente e a sustentabilidade, e até mesmo um maior ou menor conhecimento desse consumidor sobre os produtos orgânicos.

De modo geral, os alimentos orgânicos são considerados mais saudáveis, nutritivos, saborosos e seguros. Michaelidou e Hassan (2008) destacam que o consumo desses produtos tende a melhorar ou manter a saúde e a qualidade de vida dos indivíduos, o que pode influenciar a intenção de consumi-los. A qualidade dos produtos orgânicos pode ser percebida pelo consumidor não apenas por seus aspectos físicos e tangíveis, mas também pelas atitudes abstratas favoráveis (Bagozzi, 1981) a tais alimentos, ou seja, a crença de que trarão uma melhor qualidade de vida, estando diretamente ligados com a preocupação com a saúde (Hoppe et al., 2012; Olson, 2017). Zamberlan et al. (2010) concluíram em seu estudo que os consumidores brasileiros compram alimentos orgânicos, principalmente devido ao não uso de agrotóxicos, às práticas de preservação

Revista de Gestão Social e Ambiental - RGSA, São Paulo, v. 14, n. 2, p. 48-64, maio/ago. 2020. 
do ambiente e as suas características sensoriais (sabor, cor, cheiro), percebendo-os como melhores do que os alimentos convencionais. Assim, propõe-se a seguinte hipótese:

H1: A qualidade do produto percebida pelo consumidor influencia positivamente a sua atitude com relação aos alimentos orgânicos.

De acordo com Engel et al. (2000), a preocupação com o meio ambiente vem crescendo e se tornando um dos principais aspectos levados em conta no processo de compra, causando uma mudança no comportamento de parte dos consumidores que passaram a conferir informações ambientais, especialmente aquelas presentes nas embalagens dos produtos ou comentadas pelas mais diversas mídias. Bashaa et al. (2015) acrescentam que os cuidados ambientais são considerados como importantes fatores motivacionais para comprar qualquer tipo de produto, em especial os produtos orgânicos, onde a produção causa menos danos ao meio ambiente.

Nesse sentido, alguns movimentos de resistência ou anticonsumo vêm se tornando mais populares ao buscarem novas formas de consumo que estariam mais de acordo com uma necessária consciência ecológica e social (Vaccari, Cohen, \& Rocha, 2016). Assim, fatores ligados à forma de produção orgânica, sem uso de agrotóxicos ou modificações genéticas, atuariam como impulsionadores da expansão deste mercado, por meio do auxílio ao pequeno produtor, da criação de relacionamentos entre produtores e consumidores, e à proteção do meio ambiente (Dias et al., 2015; Rana \& Paul, 2017). Barcelos et al. (2015) complementam que consumidores conscientes apresentam fortes valores coletivistas e atitudes positivas em relação ao meio ambiente e à natureza, ou seja, que o consumo de orgânicos é precedido por uma atitude positiva em relação a questões ambientais e aos fatores ligados a essa forma de produção. Assim, propõe-se a seguinte hipótese:

H2: A consciência ecológica dos consumidores influencia positivamente a sua atitude com relação aos alimentos orgânicos.

Segundo Engel et al. (2000, p. 139), “[...]o conhecimento pode ter vários efeitos no uso de critérios de avaliação pelos consumidores". O indivíduo que conhece o produto pode realizar comparações para definir qual alternativa deve escolher, diferentemente do indivíduo que o desconhece e que, portanto, não terá o embasamento necessário para realizar uma escolha segura no momento da compra. Conforme Rana e Paul (2017), ao conhecerem os produtos orgânicos, os consumidores podem comparar e diferenciar a sua qualidade frente aos convencionais, de maneira a formar atitudes positivas quanto a esses produtos.

Aschemann-Witzel e Zielke (2017) também concluíram que quanto maior a consciência e o conhecimento adicional sobre alimentos orgânicos, maior é a influência positiva sobre as atitudes relacionadas aos alimentos orgânicos. Nesse sentido, torna-se essencial a presença de informações nutricionais ou de benefícios à saúde disponibilizados nos rótulos dos produtos (Cardoso \& Rodrigues, 2015). Complementarmente, Sampaio e Gosling (2014) destacam que, além do conhecimento do consumidor, é importante que as pessoas que atuam em organizações que ofertem produtos orgânicos conheçam o conceito, as características e o respectivo local de origem dos produtos. Desta forma, propõe-se a seguinte hipótese:

H3: O conhecimento dos consumidores sobre o produto influencia positivamente a sua atitude com relação aos alimentos orgânicos.

Bashaa et al. (2015) apontam que a atitude contribui bastante para explicar o comportamento de compra dos indivíduos e que, conforme a conscientização sobre os produtos orgânicos aumenta, a atitude dos consumidores torna-se cada vez mais positiva quanto ao seu consumo. Nesse sentido, atitudes positivas em relação a estes produtos influenciariam a intenção de consumi-los (Hoppe et al., 2012) e esta, por sua vez, impactaria no comportamento de compra e de consumo desses alimentos. Ainda que, no contexto da intenção de compra de alimentos orgânicos a 
influência da atitude seja inconclusiva (Chekima et al., 2019), uma atitude positiva em relação aos produtos sustentáveis - considerando tanto os atributos do produto quanto os seus aspectos ambientais - é um bom ponto de partida para estimular o consumo sustentável. Portanto, propõe-se a seguinte hipótese:

H4: A atitude dos consumidores com relação aos alimentos orgânicos influencia positivamente o consumo desses produtos.

Ainda de acordo com Engel et al. (2000), o preço é um dos critérios de avaliação mais importantes na aquisição de qualquer produto, sendo a sensibilidade do consumidor quanto ao preço um aspecto bastante relevante para a segmentação de mercado. Entretanto, nem sempre os consumidores estão buscando o preço mais baixo, às vezes a conveniência, o tipo de produto e até mesmo a sua marca podem ser mais importantes para o consumidor. Em relação aos alimentos orgânicos, Sampaio e Gosling (2014) concluíram que o preço pode ser um entrave para a efetivação da compra desses produtos, fazendo com que, em alguns casos, o consumidor opte pelos alimentos convencionais; isto se deve principalmente ao fato de que, normalmente, os alimentos orgânicos possuem um preço mais elevado quando comparados aos convencionais (Zamberlan et al., 2010).

Oluwoye et al. (2017) também destacaram, em seu estudo, que a intenção de compra dos alimentos orgânicos é maior quando a diferença entre os preços dos alimentos convencionais e orgânicos é menor. No entanto, em muitos casos, os consumidores de produtos orgânicos estão dispostos a desembolsar um valor maior para comprá-los, pois entendem que estes produtos são de melhor qualidade, sendo estes consumidores menos sensíveis a preços e mais preocupados com a qualidade do produto (Bashaa et al., 2015). Logo, pode-se entender que o preço apresenta um papel fundamental no que tange à efetivação da compra, podendo ser uma barreira ou um mecanismo de diferenciação, dependendo de como os consumidores percebem o valor do produto a ser adquirido (Aschemann-Witzel \& Zielke, 2017). Assim, propõe-se a seguinte hipótese:

H5: O preço favorável percebido pelo consumidor influencia positivamente o consumo de alimentos orgânicos.

Para Paul e Rana (2012), a disponibilidade do produto também é um dos principais atributos que motivam o processo de compra. Com o aumento da popularidade dos alimentos orgânicos, muitos empresários procuraram aumentar a disponibilidade desses produtos nos mais diferentes estabelecimentos, reservando seções e corredores exclusivos para estes itens. O aumento da oferta de alimentos orgânicos nas prateleiras, seja em termos de diversidade de produtos ou de locais, permite um maior acesso, por parte dos consumidores, a este tipo de alimento (Dettmann \& Dimitri, 2010).

No Brasil, os alimentos orgânicos passam por grande expansão, saindo do ambiente exclusivo das feiras livres e entrando nas prateleiras e gôndolas dos supermercados, sendo um dos principais meios de distribuição deste tipo de produto, já que em alguns locais existe a dificuldade de encontrá-los (Zamberlan et al., 2010). A disponibilidade desses alimentos se configura, portanto, como uma variável que pode influenciar diretamente na intenção de compra dos consumidores. Por outro lado, a falta de disponibilidade dos produtos nas lojas e a realização de esforços extras (incluindo tempo e dinheiro) para encontrá-los podem ser consideradas barreiras na intenção de compra de produtos orgânicos (Hoppe et al., 2012; Rana \& Paul, 2017). Assim, propõe-se a seguinte hipótese:

H6: A disponibilidade do produto percebida pelo consumidor influencia positivamente o consumo de alimentos orgânicos. 
Complementarmente, o preço e a disponibilidade dos produtos orgânicos são testados nesta pesquisa como variáveis moderadoras entre a atitude em relação aos alimentos orgânicos e o consumo. Assim, espera-se que o consumidor que possui uma atitude favorável ao consumo de orgânicos, a maior ou menor disponibilidade desses produtos influenciará o seu consumo de forma mais ou menos intensa. O mesmo deve ocorrer em relação ao preço dos alimentos orgânicos, ou seja, quando o consumidor apresenta uma atitude favorável em relação a esses produtos, e o seu preço é percebido como mais atrativo, maior será o consumo desses alimentos. Com base no exposto acima, propõem-se as seguintes hipóteses:

H7: A relação positiva entre a atitude do consumidor quanto aos alimentos orgânicos e o seu consumo será mais forte quanto mais favorável for o preço percebido pelo consumidor; e

H8: A relação positiva entre a atitude do consumidor quanto aos alimentos orgânicos e o seu consumo será mais forte quanto maior for a sua disponibilidade.

A Figura 1 ilustra o modelo conceitual da pesquisa, em que o consumo de alimentos orgânicos é influenciado diretamente pela atitude dos consumidores em relação a esse tipo de alimento, a qual é impactada pela consciência ecológica do consumidor, pelo conhecimento que tem sobre os produtos orgânicos e pela sua qualidade percebida. $\mathrm{O}$ consumo ainda é influenciado pelo preço e pela disponibilidade dos produtos, tanto direta quanto indiretamente, ao agirem como variáveis moderadoras entre a atitude frente aos alimentos orgânicos e o seu consumo. Algumas características pessoais, tais como idade, escolaridade, renda e hábitos de consumo, também podem ajudar a explicar o comportamento de compra desses alimentos, tanto em termos de quantidade de produtos consumidos quanto de gastos mensais. Assim, tais características foram incluídas no modelo conceitual da pesquisa como variáveis de controle.

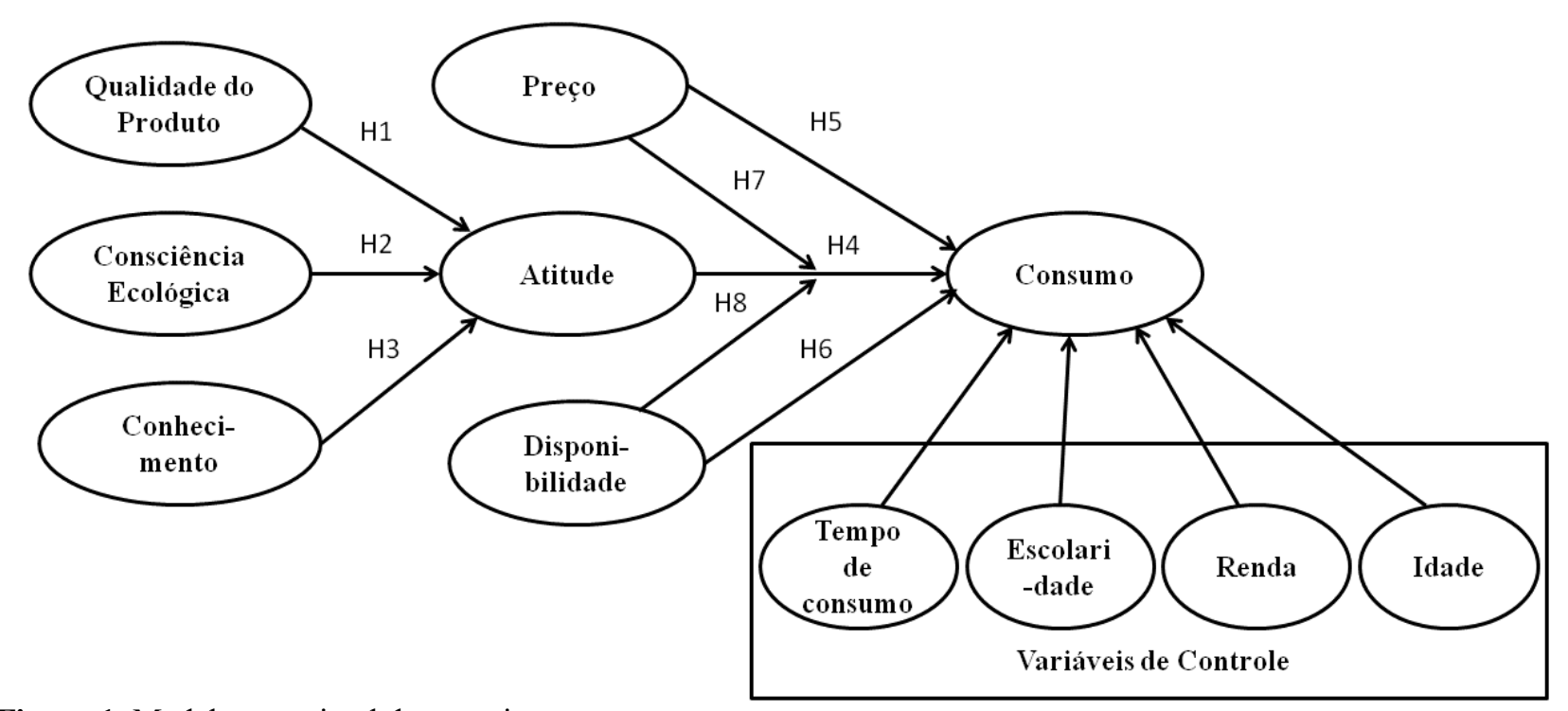

Figura 1. Modelo conceitual da pesquisa

Fonte: Elaboração própria

\section{METODOLOGIA}

Esse estudo caracteriza-se como uma pesquisa quantitativa de caráter descritivo, realizada com 219 consumidores de alimentos orgânicos participantes de grupos e comunidades interessados em produtos orgânicos, presentes na rede social Facebook. Esta foi escolhida para alcançar um maior número de respondentes, visto que é considerada a plataforma com maior número de usuários 
ativos no mundo (Imme, 2020). A amostra classifica-se como não probabilística, sendo os respondentes selecionados por conveniência. A pesquisa partiu da elaboração de um questionário estruturado, composto por questões fechadas, e desenvolvido com base nos construtos presentes no modelo conceitual.

O questionário foi composto por três seções: a primeira, tratando dos hábitos de consumo de alimentos orgânicos (incluindo o tempo que o respondente consome tais produtos, a frequência de consumo, o gasto mensal com alimentos orgânicos, os tipos de alimentos consumidos e o local onde compra); a segunda, abordando diferentes aspectos associados aos alimentos orgânicos (incluindo questões sobre disponibilidade, conhecimento, consciência ecológica, qualidade do produto, preço e atitude dos consumidores com relação aos alimentos orgânicos) e a terceira, identificando o perfil sociodemográfico dos respondentes (incluindo algumas questões, tais como sexo, idade, estado civil, escolaridade e renda mensal familiar).

Com relação à segunda seção do questionário, esta foi formada por questões adaptadas de outros instrumentos já validados e testados empiricamente em estudos anteriores, sendo operacionalizadas em uma escala Likert de cinco pontos, variando de (1) discordo totalmente a (5) concordo totalmente. O consumo de alimentos orgânicos foi mensurado por meio de dois itens: quantidade de produtos orgânicos consumidos mensalmente e gasto mensal com a compra de alimentos orgânicos. Ao modelo, foram incluídas a idade, a renda e a escolaridade do respondente como variáveis de controle, assim como o tempo que o mesmo consome produtos orgânicos. O instrumento aplicado está disponível no final do artigo (quadro itens de instrumento) com as suas referências e estatísticas descritivas.

Após a elaboração do instrumento de coleta de dados e sua aplicação, procedeu-se à etapa de análise, por meio de estatísticas descritivas e da técnica de modelagem de equações estruturais, realizada por intermédio do software SmartPLS 3.0 (Partial Least Squares). Do total de 228 respostas retornadas, foram consideradas válidas 219, sendo eliminados nove questionários por terem sido preenchidos de forma incorreta, estando parcialmente incompletos ou sem preenchimento.

Verificada a qualidade dos dados coletados, procedeu-se a etapa de modelagem de equações estruturais. Com base nesse método, é realizada a avaliação do modelo de mensuração e do modelo estrutural, de forma a se obter medidas confiáveis para a interpretação das relações de causalidade propostas. Primeiramente, analisou-se o modelo de mensuração, o qual busca analisar a relação entre os construtos latentes e os itens relacionados a cada um deles. Os construtos foram verificados quanto a sua validade convergente e discriminante por meio da análise fatorial confirmatória (AFC). Uma das formas de se analisar a validade discriminante dos construtos é por meio do critério das cargas cruzadas, que ocorre quando os itens apresentam maiores cargas fatoriais no seu próprio construto em comparação com os demais (Chin, 1998). Essa análise sugeriu a exclusão de cinco questões, as quais apresentaram cargas fatoriais inferiores a $0,70 \mathrm{em}$ seus respectivos constructos. A Tabela 1 destaca as cargas fatoriais de todos os itens, os quais apresentaram valores superiores nos seus respectivos construtos e menores nos demais, confirmando assim a validade discriminante.

Também foi avaliada a validade convergente dos construtos, por meio do valor das cargas fatoriais e pelo critério da variância média esperada (do inglês, average variance expected - AVE), cujos valores excederam o limite mínimo de 0,50 (Tabela 2). Tanto as cargas fatoriais quanto os valores da AVE servem de base para assegurar que os construtos do modelo proposto demonstram validade convergente. A validade discriminante ainda foi verificada pelo critério de FornellLarcker, em que a raiz quadrada da AVE deve ser maior que as correlações entre os construtos do modelo (destacado em negrito na diagonal). 
Tabela 1: Análise Fatorial Confirmatória

\begin{tabular}{lccccccc}
\hline \multicolumn{1}{c}{ Itens } & Atitude & Conhecimento & Consciência & Disponibilidade & Preço & Qualidade & Consumo \\
\hline Atitude2 & $\mathbf{0 , 9 5 4}$ & 0,335 & 0,577 & 0,034 & 0,436 & 0,644 & 0,273 \\
Atitude3 & $\mathbf{0 , 9 6 2}$ & 0,353 & 0,587 & 0,010 & 0,446 & 0,630 & 0,249 \\
Atitude4 & $\mathbf{0 , 9 4 8}$ & 0,400 & 0,619 & 0,035 & 0,492 & 0,653 & 0,300 \\
Conh1 & 0,314 & $\mathbf{0 , 8 8 0}$ & 0,409 & 0,226 & 0,333 & 0,319 & 0,352 \\
Conh2 & 0,284 & $\mathbf{0 , 8 3 0}$ & 0,362 & 0,248 & 0,307 & 0,220 & 0,317 \\
Conh3 & 0,345 & $\mathbf{0 , 7 8 6}$ & 0,419 & 0,268 & 0,244 & 0,287 & 0,388 \\
Cons2 & 0,426 & 0,239 & $\mathbf{0 , 7 3 8}$ & 0,096 & 0,354 & 0,548 & 0,154 \\
Cons3 & 0,563 & 0,507 & $\mathbf{0 , 8 0 1}$ & 0,162 & 0,333 & 0,566 & 0,325 \\
Cons4 & 0,423 & 0,319 & $\mathbf{0 , 7 6 1}$ & 0,131 & 0,455 & 0,666 & 0,105 \\
Disp1 & $-0,044$ & 0,104 & $-0,011$ & $\mathbf{0 , 7 4 0}$ & 0,082 & $-0,059$ & 0,225 \\
Disp2 & 0,071 & 0,287 & 0,252 & $\mathbf{0 , 7 0 4}$ & 0,305 & 0,178 & 0,174 \\
Disp3 & 0,045 & 0,295 & 0,174 & $\mathbf{0 , 8 1 4}$ & 0,312 & 0,074 & 0,252 \\
Prec1 & 0,482 & 0,372 & 0,532 & 0,182 & $\mathbf{0 , 7 8 1}$ & 0,544 & 0,255 \\
Prec2 & 0,341 & 0,294 & 0,390 & 0,285 & $\mathbf{0 , 8 4 8}$ & 0,328 & 0,182 \\
Prec3 & 0,360 & 0,251 & 0,342 & 0,282 & $\mathbf{0 , 8 5 3}$ & 0,344 & 0,241 \\
Prec4 & 0,394 & 0,241 & 0,336 & 0,274 & $\mathbf{0 , 8 5 2}$ & 0,321 & 0,204 \\
Qual1 & 0,546 & 0,253 & 0,629 & 0,045 & 0,386 & $\mathbf{0 , 8 4 2}$ & 0,150 \\
Qual3 & 0,593 & 0,263 & 0,707 & 0,052 & 0,386 & $\mathbf{0 , 9 0 4}$ & 0,151 \\
Qual4 & 0,637 & 0,362 & 0,692 & 0,094 & 0,476 & $\mathbf{0 , 9 0 0}$ & 0,213 \\
Consumo1 & 0,266 & 0,414 & 0,223 & 0,265 & 0,198 & 0,168 & $\mathbf{0 , 8 6 5}$ \\
Consumo2 & 0,217 & 0,300 & 0,232 & 0,226 & 0,263 & 0,163 & $\mathbf{0 , 8 2 2}$ \\
\hline Fonte: Elab & & & & &
\end{tabular}

Fonte: Elaboração própria

Avaliou-se a fidedignidade das escalas por meio da confiabilidade composta (do inglês, Composite Reliability - CR), como indicado na Tabela 2. A confiabilidade composta é preferível ao alfa de Cronbach porque oferece uma melhor estimativa da variância compartilhada pelos seus indicadores, além de utilizar as cargas dos itens obtidos em uma rede nomológica, diferentemente do alfa de Cronbach (Hair, Sarstedt, Hopkins, \& Kuppelwieser, 2014). Os escores da confiabilidade composta de todos os construtos excederam o limite mínimo de 0,70, indicando uma boa confiabilidade das escalas.

Tabela 2: Variância compartilhada, correlações e confiabilidade dos construtos

\begin{tabular}{lcccccccccc}
\hline & Média & CR & AVE & Atitude & Conhec & Consc & Consumo & Disp & Preço & Qualid \\
\hline Atitude & 4,09 &, 97 &, 91 &, 96 & & & & & & \\
Conhecimento & 3,35 &, 87 &, 69 &, 38 &, 83 & & & & & \\
Consciência & 4,14 &, 81 &, 59 &, 62 &, 48 &, 77 & & & \\
Consumo & 2,95 &, 83 &, 71 &, 29 &, 43 &, 27 &, 84 & & & \\
Disponibilidade & 3,13 &, 80 &, 57 &, 03 &, 30 &, 17 &, 29 &, 75 &, 83 &, 80 \\
Preço & 3,28 &, 90 &, 70 &, 48 &, 35 &, 49 &, 27 &, 30 &, 83 &, 47 \\
Qualidade & 4,36 &, 91 &, 78 &, 67 &, 33 &, 77 &, 20 &, 07 &, 47 & \\
\hline
\end{tabular}

Fonte: Elaboração própria

\section{RESULTADOS}

Do total de respondentes, $150(68,5 \%)$ eram do sexo feminino e $68(31,1 \%)$ masculino, um dos respondentes deixou a questão em branco. Quanto ao estado civil, 158 (72,1\%) declararam-se solteiros e $52(23,7 \%)$ casados. No que se refere à idade dos respondentes, estes se encontram entre 18 e 72 anos, sendo a média de idade igual a 31 anos ( $\pm 12,2$ anos). Quanto à escolaridade, todos respondentes possuem pelo menos o Ensino Médio completo, dos quais 59,8\% estão cursando ou já concluíram o Ensino Superior, enquanto 40,2\% cursam ou já concluíram alguma pós-graduação. Estes dados são similares a outros estudos realizados no Brasil e em outros países que analisaram o perfil do consumidor de produtos orgânicos (Dettmann \& Dimitri, 2010; Nasir \& Karakaya, 2014; Sampaio \& Gosling, 2014; Thøgersen et al., 2015). 
No que confere à renda mensal familiar, $22,8 \%$ dos respondentes recebem até dois salários mínimos, $23,3 \%$ entre dois e quatro salários e 53,9\% ganham mais do que quatro salários. Referente ao tempo que consomem alimentos orgânicos, 30,6\% dos respondentes afirmaram que consomem há um ano ou menos, $22,8 \%$ há pelo menos dois anos, $10,5 \%$ há pelo menos três anos e $35,6 \%$ há quatro anos ou mais. Quando questionados sobre a frequência com que consomem alimentos orgânicos, 48,4\% declararam consumir várias vezes na semana; 20,1\%, uma vez por semana; $17,8 \%$, duas ou três vezes por mês e $13,7 \%$, uma ou duas vezes nos últimos meses. Esses resultados denotam uma alta frequência no consumo de alimentos orgânicos por parte dos consumidores participantes do estudo. No entanto, os dados referentes aos gastos mensais com esses alimentos demonstram um predomínio dos gastos inferiores a $\mathrm{R} \$ 60,00$ por mês, representando $55,7 \%$ ( $\mathrm{n}=$ 122) dos casos.

Além de hortaliças (citadas por 173 respondentes, 79,0\%), frutas (166 respondentes, 75,8\%) e cereais (85 respondentes, 38,8\%) aparecem como os tipos de alimentos orgânicos mais consumidos, sendo citados também mel, suco, bolo, biscoito, pão, café, erva mate, arroz, feijão, açúcar, sal, castanha, vinho, óleo, farinha, grãos e ovos. Com relação ao local em que adquirem os alimentos orgânicos, os locais mais citados foram a feira $(63,9 \%)$, o supermercado $(38,8 \%)$, a feira ecológica $(34,2 \%)$, o plantio próprio $(24,7 \%)$ e a fruteira $(22,4 \%)$. Em relação ao preço dos alimentos orgânicos, identificou-se que a maioria dos respondentes $(72,2 \%)$ os consideram mais caros ou muito mais caros que os convencionais, $23,3 \%$ acreditam ser quase o mesmo preço e apenas $4,6 \%$ julgam ser mais baratos.

Em seguida, buscando-se avaliar o relacionamento preditivo entre os construtos e testar as hipóteses do modelo proposto, analisou-se o modelo estrutural. Foi utilizada a técnica de bootstrapping com 5.000 amostras para avaliar a aderência geral do modelo, bem como de seus parâmetros. Ao analisar-se a Figura 2, percebe-se que a Qualidade do Produto $(\beta=0,48 ; p<0,001)$, a Consciência Ecológica $(\beta=0,19 ; p<0,05)$ e o Conhecimento sobre orgânicos $(\beta=0,13 ; p<0,05)$ apresentaram-se como importantes preditores da Atitude dos consumidores com relação aos alimentos orgânicos, cujas relações apresentaram efeito positivo e significativo, demonstrando um elevado grau de explicação (49,3\%) da variável dependente (Atitude), confirmando as hipóteses H1, $\mathrm{H} 2$ e H3.

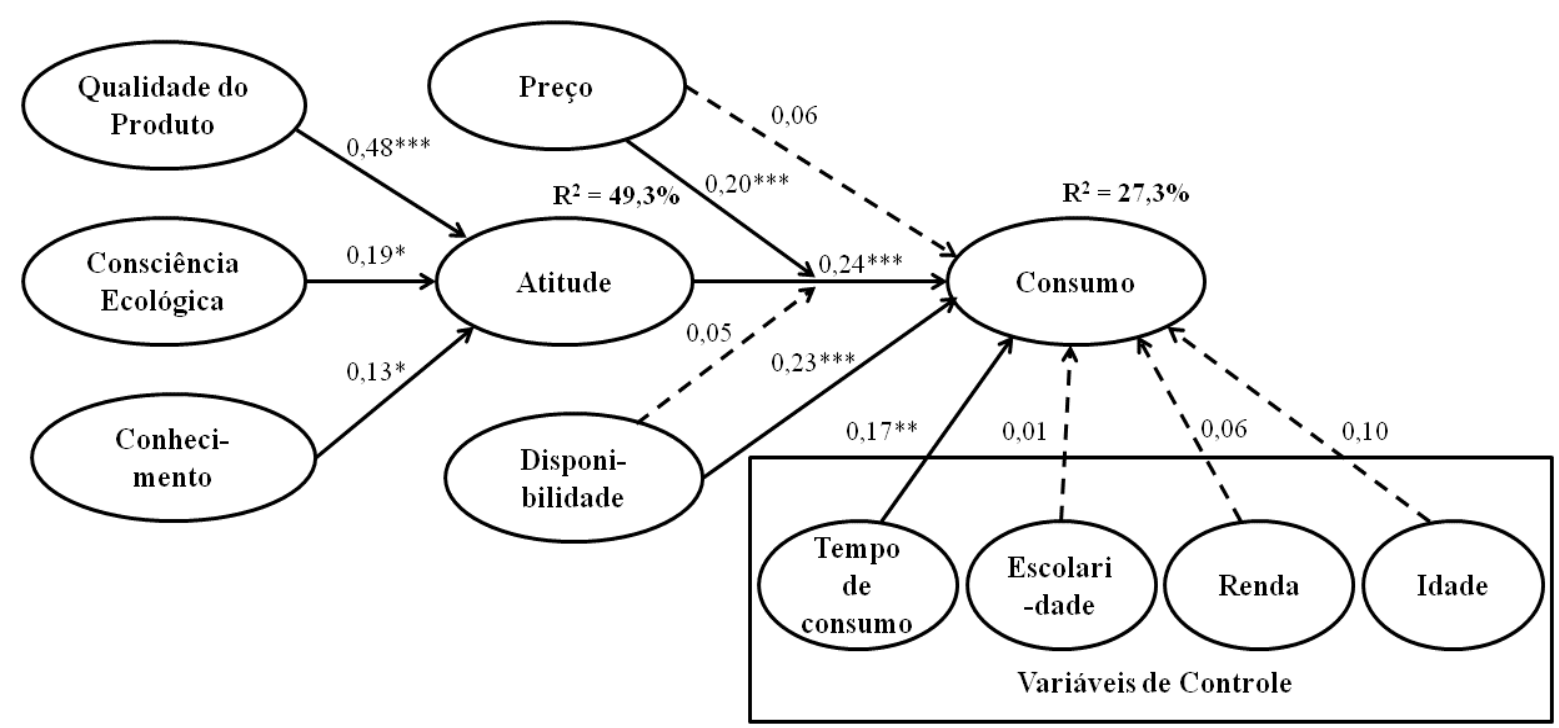

Figura 2. Modelo Estrutural

Fonte: Elaboração própria

Complementarmente, pôde-se perceber que a atitude dos consumidores com relação aos alimentos orgânicos $(\beta=0,24 ; p<0,001)$ e a disponibilidade do produto $(\beta=0,23 ; p<0,001)$ também apresentaram correlações significativas e positivas com o consumo desses alimentos. $\mathrm{O}$ 
preço, por outro lado, não confirmou um efeito direto e significativo no consumo de orgânicos $(\beta=$ 0,06; $p>0,05)$; entretanto, quando analisado em conjunto com a atitude dos consumidores, pôde-se perceber a presença de um efeito interativo positivo e significativo $(\beta=0,20 ; p<0,001)$, sugerindo que quanto melhor for o preço desses produtos para o consumidor, cuja atitude em relação a eles seja favorável, maior será o seu consumo - confirmando-se, portanto, as hipóteses H4, H6 e H7.

Percebe-se que uma atitude mais favorável dos consumidores interessados neste movimento está mais fortemente associada a sua exigência pela qualidade dos produtos que consomem, pelos impactos da agricultura sobre o meio ambiente e produtores locais, bem como pelo nível de conhecimento que possuem sobre os alimentos orgânicos. Ou seja, o fato de os consumidores conhecerem e reconhecerem os benefícios destes produtos, sejam eles para a sua saúde, a de sua família ou ainda para o bem da sociedade, podem ser apontadas como fortes razões que causam mudanças de atitude dos consumidores em relação aos alimentos orgânicos. Nesse sentido, identificou-se que a percepção dos consumidores quanto à qualidade desses produtos, entendidos como mais saudáveis, com melhor aroma e sabor que os convencionais, destaca-se como o principal desencadeador de uma atitude mais favorável com relação ao consumo de tais produtos. Bezawada e Pauwels (2013) também identificaram que os atributos sabor e saúde são determinantes na aquisição dos alimentos orgânicos.

Além da qualidade do produto, outros fatores que contribuem significativamente para uma mudança de atitude dos consumidores frente aos alimentos orgânicos, ainda que em menor intensidade, são a consciência ecológica, a qual retrata a percepção do consumidor quanto ao auxílio oportunizado ao pequeno produtor, à preocupação pela escolha de alimentos menos prejudiciais às pessoas e ao meio ambiente, cuja escolha de compra é influenciada por razões ecológicas; e um maior conhecimento sobre os benefícios dos alimentos orgânicos e da sua forma de produção. $\mathrm{O}$ auxílio ao pequeno produtor ou a produtores locais tem sido apontado em alguns estudos recentes (Olson, 2017; Rana \& Paul, 2017; Chekima et al., 2019) como um importante influenciador do consumo de orgânicos. Barcelos et al. (2015) defendem que os consumidores desses produtos possuem valores coletivos mais intensos e, portanto, apresentam efeito positivo e significativo na sua atitude em relação ao meio ambiente e à natureza, a qual pode desencadear um aumento no consumo de produtos orgânicos. Ainda, a presença de rótulos de alimentos embalados contendo informações nutricionais e de saúde também influencia a atitude e as intenções de compra dos consumidores, conforme destacam Kozup, Creyer e Burton (2003). Segundo os autores, em geral, os consumidores têm uma atitude mais favorável em relação aos produtos que apresentam descrições detalhadas nos seus rótulos, incluindo informações nutricionais, proveniência do produto e etc. Esses esforços tornam os consumidores de hoje mais conscientes e preocupados com um estilo de vida saudável.

Da mesma forma, identificou-se que quanto maior for a disponibilidade dos alimentos orgânicos em termos de acesso e distribuição, maior será o seu consumo. Este resultado corrobora com a grande maioria dos trabalhos realizados anteriormente que cita a disponibilidade como um fator influenciador da intenção e do consumo de orgânicos, já que estes produtos só podem ser adquiridos se estiverem disponíveis aos seus potenciais compradores (Hoppe et al., 2012; Nasir \& Karakaya, 2014; Rana \& Paul, 2017). A entrada dos alimentos orgânicos em locais convencionais e, particularmente, nas gôndolas dos supermercados, embora nem sempre seja vista como um desenvolvimento desejável por parte do movimento de agricultura orgânica (devido à necessidade de uma produção em maior escala, padronizada, e que estaria elitizando o consumo e dividindo os lucros dos seus produtores), tem facilitado o acesso a este tipo de produto, cuja disponibilidade sempre foi apontada como um dos principais fatores críticos para torná-lo popular entre os consumidores.

Ainda assim, alguns estudos têm destacado que os alimentos orgânicos comercializados de forma in natura enfrentam uma série de obstáculos ao entrarem em grandes redes de supermercados, sendo a sua alta perecibilidade o principal fator, pois implica em um maior volume de perdas, na necessidade de maior frequência de entrega e consequente necessidade de uma maior 
capacidade logística e de produção por parte dos agricultores, de modo que a viabilize economicamente. Esses fatores acabariam excluindo pequenos produtores deste mercado, abrindo espaço para empresas distribuidoras que articulem melhor essa relação produtor-supermercado - o que talvez justifique a não confirmação da hipótese $\mathrm{H} 8$, de que uma maior disponibilidade de alimentos orgânicos poderia incentivar o consumidor que possui uma atitude favorável a estes produtos a aumentar o seu consumo. Outra opção seria a de manter a produção e o consumo de orgânicos em pequena escala, em mercados locais, como as feiras livres ou de produtores de orgânicos - isso por oportunizarem a aquisição de vegetais frescos ou por contornarem o problema das perdas devido à perecibilidade destes produtos, uma vez que a oferta é ajustada à demanda sem existência de um tempo de permanência nas bancas, evitando custos assumidos integralmente pelos produtores quando da comercialização em supermercados (Guivant, 2003).

Com relação ao preço dos alimentos orgânicos, identificou-se que ele diretamente não influencia o comportamento de compra do consumidor. Entretanto, com consumidores cuja atitude em relação aos alimentos orgânicos é favorável, à medida que o preço desses produtos é considerado aceitável, justo e com uma boa relação custo-benefício, este influencia de forma positiva o comportamento de consumo desses produtos. Hoppe et al. (2013) identificaram que os consumidores de orgânicos percebem as suas vantagens relativas, mesmo quando são comparadas as diferenças com os alimentos convencionais, sugerindo que estes indivíduos são menos influenciáveis pelo custo e risco percebidos na compra de alimentos orgânicos, por acreditarem que os seus benefícios explicam de forma mais apropriada as suas atitudes individuais. Essa mesma relação também aparece no trabalho de Figueiró et al. (2012), em que foi observado que os entrevistados estariam dispostos a pagar um preço mais elevado pelos orgânicos, pois teriam como contrapartida alimentos de melhor qualidade, sem agrotóxicos, que beneficiam a saúde pessoal e a natureza, e que valoriza os seus produtores.

Esse bem estar proporcionado ao consumidor interfere na sua percepção de custo-benefício, explicando porque os consumidores de orgânicos, em geral, estão dispostos a pagar um preço mais elevado, tendo consciência de que as vantagens proporcionadas valem a pena. Aschemann-Witzel e Zielke (2017), entretanto, sugerem que o preço dos alimentos orgânicos não deve exceder a $30 \%$ do valor cobrado pelos produtos convencionais. Considerando que existe uma expectativa futura de diminuição de preços, seja pelo aumento da produção e dos canais de distribuição e venda (Bezawada \& Pauwels, 2013), essa possibilidade não seria tão irreal.

Por fim, ao se analisarem algumas características pessoais e hábitos de consumo (tratadas aqui como variáveis de controle), apenas o tempo que o consumidor consome alimentos orgânicos apresentou efeito positivo significativo $(\beta=0,17 ; p<0,01)$ sobre o consumo de tais alimentos. Conforme destacam Hoppe et al. (2012), quanto maior a experiência prévia do consumidor com alimentos orgânicos, maior será a sua intenção de continuar comprando tais alimentos - o mesmo foi identificado neste estudo, entretanto, relacionando-se essa experiência prévia com o comportamento real de consumo, e não de intenção. Percebe-se que o fato de o indivíduo já estar consumindo alimentos orgânicos por um período maior acaba por criar um envolvimento dessas pessoas com tais produtos, tornando-se um hábito, uma rotina de alimentação e, assim, criando certa fidelidade com este tipo de produto, o que se reflete em uma maior variedade de produtos consumidos e de valores gastos pelos consumidores e famílias com alimentos orgânicos. Chekima et al. (2017) ressaltam que os alimentos são produtos consumidos diariamente, o que significa que o consumo recorrente de produtos, como os alimentos orgânicos, aparece como um forte e sólido preditor do comportamento recorrente de compra.

Zamberlan et al. (2010) afirmam que as pessoas que adotam uma alimentação orgânica tendem a inserir tais produtos em sua dieta com considerável intensidade, visto que os resultados de sua pesquisa apontaram que $77,3 \%$ dos respondentes afirmaram consumir alimentos orgânicos frequentemente, enquanto apenas $22,7 \%$ afirmaram fazê-lo raramente. Estes consumidores habituais são mais conscientes em termos éticos, levando mais em consideração os valores da sociedade e 
valorizando o meio ambiente natural (Hoppe et al., 2013). Já as demais variáveis de controle analisadas (escolaridade, renda e idade), aparentemente, não estão associadas a um maior consumo de alimentos orgânicos por parte dos consumidores pesquisados. Segundo Aschemann-Witzel e Zielke (2017), as variáveis sociodemográficas são geralmente menos úteis como preditoras do que as variáveis psicográficas (como crenças, atitudes e comportamentos), o que pode justificar a ausência de tais relações.

Com relação ao consumo de alimentos orgânicos, o modelo apresentou um moderado grau de explicação $(27,3 \%)$ da variável dependente, sugerindo que a atitude dos consumidores com relação aos alimentos orgânicos, a disponibilidade do produto, o tempo que estes produtos são consumidos pelas pessoas e o preço pago pelos consumidores (este último, de forma indireta) influenciam significativamente o seu consumo. Cabe destacar que estas quatro relações exercem pesos muito semelhantes como variáveis preditoras do consumo desses alimentos (cujo $r$ variou entre 0,17 e 0,24 ), tanto em termos de variedade de produtos consumidos quanto de valores mensais gastos.

\section{CONSIDERAÇÕES FINAIS}

Este trabalho analisou diferentes determinantes do comportamento do consumidor de alimentos orgânicos, por meio da proposição de um modelo estrutural capaz de explicar e prever fatores que influenciam o consumo efetivo destes produtos. Para isso, realizou-se uma pesquisa survey, junto a 219 consumidores de alimentos orgânicos, em que foram analisadas a influência direta e indireta de cinco fatores associados à atitude e ao comportamento do consumidor em relação ao consumo de produtos orgânicos, sendo eles: a consciência ecológica, o conhecimento sobre o produto, a qualidade do produto, o preço e a sua disponibilidade. Analisou-se, ainda, um efeito moderador do preço e da disponibilidade dos alimentos orgânicos na relação entre a atitude dos consumidores e o seu comportamento de compra, juntamente com algumas características pessoais e hábitos de consumo.

Verificou-se que a qualidade do produto, a consciência ecológica e o conhecimento sobre os alimentos orgânicos influenciam positivamente a atitude dos consumidores em relação aos produtos orgânicos, sendo a qualidade do produto o principal preditor. Nota-se que a qualidade do alimento orgânico está associada a uma maior preocupação com a saúde, sendo percebida pelos consumidores desses produtos como melhores do que os alimentos convencionais, sendo considerados mais saudáveis, nutritivos e saborosos. Identificou-se, também, que o fato de a produção de alimentos orgânicos beneficiar o pequeno produtor e ser menos prejudicial às pessoas e ao meio ambiente influencia positivamente a atitude dos consumidores, levando-os a consumir com maior frequência tais alimentos.

Confirmou-se, ainda, que a atitude do consumidor, a disponibilidade do produto, o tempo em que estes alimentos são consumidos e o preço percebido pelos consumidores pró-orgânicos apresenta efeito significativo no seu consumo, tanto em termos de variedade de produtos consumidos como em valores gastos mensalmente. Isso sugere que quanto mais habitual se torna o consumo de alimentos orgânicos, seja pelas crenças do consumidor, pela facilidade de encontrá-los no mercado ou ainda pelo preço percebido como justo, mais frequente será o seu consumo e sua compra.

A partir dos resultados aqui obtidos, pode-se sugerir aos produtores e vendedores de alimentos orgânicos que reforcem as propriedades referentes a sua qualidade, como os benefícios proporcionados à saúde, a produção livre de agrotóxicos, o melhor sabor e a presença de nutrientes. Podem, ainda, explorar a consciência ecológica dos consumidores como fonte de conteúdo para promoção desses produtos, enfatizando características que justifiquem o seu preço (são mais saudáveis e melhores que os convencionais, são menos prejudiciais às pessoas e ao meio ambiente, auxiliam o pequeno produtor, não são produzidos em larga escala, etc.) e façam com que o consumidor perceba seu valor transacional como um preço justo e razoável. O desenvolvimento de 
campanhas de publicidade, embalagens e rótulos informando aos consumidores sobre estas propriedades e benefícios associados aos alimentos orgânicos também poderiam ser utilizadas como estratégias mercadológicas.

Como contribuições teóricas, pode-se destacar a elaboração de um modelo que avalia fatores que influenciam, de forma direta e indireta, a atitude dos consumidores frente aos produtos orgânicos e o consumo desses produtos. Dentro dos estudos sobre consumo de alimentos orgânicos, Thøgersen et al. (2017) afirmam que existe uma distinção entre aqueles que se concentram em analisar percepções sobre os produtos orgânicos e os que focam nas atitudes e nos comportamentos dos consumidores de orgânicos. Desta forma, este trabalho contribui para a literatura ao aliar as duas vertentes, as percepções sobre os atributos dos produtos orgânicos, e as atitudes e comportamentos de compra dos consumidores desses produtos. Outra contribuição refere-se ao resgate da literatura nacional e internacional sobre o comportamento do consumidor de alimentos orgânicos e os elementos influenciadores deste consumo no contexto nacional, podendo auxiliar outros pesquisadores interessados neste tema de pesquisa.

Como limitações do estudo, ressalta-se que a pesquisa atingiu - de forma intencional apenas consumidores brasileiros, podendo ocorrer variações devido às diferentes características culturais e geográficas de outros países ou regiões. Outra limitação diz respeito à forma de seleção e ao perfil dos respondentes analisados, todos membros da rede social Facebook, interessados na agricultura orgânica. Estas limitações não invalidam os resultados aqui obtidos, mas sugerem cuidados quanto a sua generalização. Por fim, sugere-se para pesquisas futuras a realização de estudos de natureza qualitativa com consumidores de alimentos orgânicos, tendo o intuito de identificar outros aspectos não estudados nesta pesquisa que ajudem a explicar melhor o comportamento de consumo destes consumidores.

\section{REFERÊNCIAS}

Aschemann-Witzel, J., \& Zielke, S. (2017). Can't buy me green? A review of consumer perceptions of and behavior toward the price of organic food. Journal of Consumer Affairs, 51(1), 211-251.

Bagozzi, R. (1981). Attitudes, intentions, and behavior: a test of some key hypotheses. Journal of Personality and Social Psychology, 41(4), 607-627.

Barcelos, M., Bossle, M., Perin, M., \& Vieira, L. (2015). Consumo de alimentos eco-inovadores: como valores e atitudes direcionam a compra dos consumidores de orgânicos? Revista Brasileira de Marketing, 14(1), 110-121.

Bashaa, M., Mason, C., Shamsudin, M., Hussain, H., \& Salem, M. (2015). Consumers attitude towards organic food. Procedia Economics and Finance, 31, 444-452.

Bezawada, R., Pauwels, K. (2013). What is special about marketing organic products? Journal of Marketing, 77(1), 31-51.

Boehm, C. (2018). Produção orgânica está em expansão no país. Recuperado em 16 de março, 2019, de http://agenciabrasil.ebc.com.br/economia/noticia/2018-08/producao-organica-esta-emexpansao-no-pais.

Cardoso, J., \& Rodrigues, L. (2015). Embalagem de alimento orgânico: signos entre identidade e convenções. Revista Fronteiras - estudos midiáticos, 17(1), 104-117.

Chekima, B., Igau, A., Wafa, S., \& Chekima, K. (2017). Narrowing the gap: factors driving organic food consumption. Journal of Cleaner Production, 166,1438-1447.

Chekima, B., Chekima, K., \& Chekima, K. (2019). Understanding factors underlying actual consumption of organic food: The moderating effect of future orientation. Food Quality and Preference, 74, 49-58. 
Chin, W. (1998). The partial least squares approach to structural equation modeling. Modern Methods for Business Research, 295(2), 295-336.

Dettmann, R., \& Dimitri, C. (2010). Who's Buying Organic Vegetables? Demographic Characteristics of U.S. Consumers. Journal of Food Products Marketing, 16(1),79-91.

Dias, V., Schultz, G., Schuster, M., Talamini, E., \& Révillion, J. (2015). O mercado de alimentos orgânicos: um panorama quantitativo e qualitativo das publicações internacionais. Ambiente \& sociedade, 18(1), 161-182.

Engel, J., Blackwell, R., \& Miniard, P. (2000). Comportamento do consumidor (8a ed). Rio de Janeiro: Livros Técnicos e Científicos Editoria S.A.

Figueiró, P., Batistella, Z., Jr., Silva, V., Saldanha, C., \& Slongo, L. (2012). Motivações e valores determinantes para o consumo de alimentos orgânicos. Anais do Encontro Nacional da Associação Nacional de Pós-Graduação e Pesquisa em Administração, Rio de Janeiro, RJ, Brasil, 36.

Grohmann, M., Battistella, L., Velter, A., \& Casasola, F. (2012). Comportamento ecologicamente consciente do consumidor: adaptação da escala ECCB para o contexto brasileiro. Revista de Gestão Social e Ambiental, 6(1), 102-116.

Guimarães, I., Defante, L., Sauer, L., \& Lima-Filho, D. (2013). Produtos orgânicos: como os consumidores os veem? Revista Brasileira de Administração Científica , 4(1), 50-61.

Guivant, J. (2003). Os supermercados na oferta de alimentos orgânicos: apelando ao estilo de vida ego-trip. Ambiente \& sociedade, 6(2), 63-81.

Hair, J., Jr, Sarstedt, M., Hopkins, L., \& Kuppelwieser, V. (2014). Partial least squares structural equation modeling (PLS-SEM) An emerging tool in business research. European Business Review, 26(2), 106-121.

Hoppe, A., Barcellos, M., Vieira, L., \& Matos, C. (2012). Comportamento do consumidor de produtos orgânicos: uma aplicação da teoria do comportamento planejado. Revista Base, 9(2), pp. 174-188.

Hoppe, A., Vieira, L., \& Barcellos, M. (2013). Consumer behaviour towards organic food in Porto Alegre: an application of the theory of planned behaviour. Revista de Economia e Sociologia Rural, 51(1), 69-90.

Imme, A. (2020). Ranking das redes sociais: as mais usadas no Brasil e no mundo, insights e materiais gratuitos. Recuperado em 13 de junho, 2020, de

https://resultadosdigitais.com.br/blog/redes-sociais-mais-usadas-no-brasil/.

Kozup, J., Creyer, E., Burton, S. (2003). Making healthful food choices: the influence of health claims and nutrition information on consumers' evaluations of packaged food products and restaurant menu items. Journal of Marketing, 67(2), 19-34.

Ministério da Agricultura, Pecuária e Abastecimento. (2015). Mercado brasileiro de orgânicos deve movimentar $R \$ 2,5$ bi em 2016. Recuperado em 30 de maio, 2016, de

http://www.agricultura.gov.br/comunicacao/noticias/2015/09/mercado-brasileiro-de-organicosdeve-movimentar-rs-2-bi-em-2016.

Michaelidou, N., \& Hassan, L. (2008). The role of health consciousness, food safety concern and ethical identity on attitudes and intentions towards organic food. International Journal of Consumer Studies, 32(2), 163-170.

Nasir, V., \& Karakaya, F. (2014). Consumer segments in organic foods market. Journal of Consumer Marketing, 31(4), 263-277. 
Comportamento do consumidor de alimentos orgânicos: um modelo baseado em influenciadores diretos e indiretos

Olson, E. (2017). The rationalization and persistence of organic food beliefs in the face of contrary evidence. Journal of Cleaner Production, 140, 1007-1013.

Oluwoye, J., Chembezi, D., \& Herbert, B. (2017). Consumer perception of organic food products and purchase behavior of shopping in outlets of retail chains: a pilot study of Huntsville, Alabama. International Journal of Agricultural Research, Sustainability, and Food Sufficiency, 4(3), 165-173.

Pato, C., \& Tamayo, A. (2006). A escala de comportamento ecológico: desenvolvimento e validação de um instrumento de medida. Revista de Psicologia, 11(3), 289-296.

Paul, J., \& Rana, J. (2012). Consumer behavior and purchase intention for organic food. Journal of Consumer Marketing, 29(6), 412-422.

Peattie, K. (2015). Sustainability marketing. In: Handbook of research on sustainable consumption. Edward Elgar Publishing.

Pereira, M., Lima-Filho, D., Maciel, W., \& Oliveira, D. (2015). Determinants of organic products consumption. Revista Brasileira de Marketing, 14(1), 122-137.

Perosa, J., Moori, R., Lombardi, M., \& Perosa, B. (2009). O estímulo local e o consumo de produtos orgânicos em Botucatu, S. P. Revista de Estudos Sociais, 2(22), Ano 11, 59-77.

Rana, J., \& Paul, J. (2017). Consumer behavior and purchase intention for organic food: A review and research agenda. Journal of Retailing and Consumer Services, 38, 157-165.

Sampaio, D., \& Gosling, A. (2014). Intenção de compra e consumo de alimentos orgânicos. Revista Gestão Organizacional, 7(1), 43-62.

Soares, F., \& Marzzaro, I. (2020). Orgânicos movimentam $R \$ 4,5$ bilhões no país e atraem número recorde de produtores. Recuperado em 20 de fevereiro, de 2020, de

https://gauchazh.clicrbs.com.br/economia/campo-e-lavoura/noticia/2020/01/organicos

movimentam-r-45-bilhoes-no-pais-e-atraem-numero-recorde-de-produtores-

ck5s854e10d2801qd635fbbuu.html

Thøgersen, J., Barcellos, M., Perin, M., \& Zhou, Y. (2015). Consumer buying motives and attitudes towards organic food in two emerging markets: China and Brazil. International Marketing Review, 32(3/4), 389-413.

Thøgersen, J., Pedersen, S., Paternoga, M., Schwendel, E., \& Aschemann-witzel, J. (2017). How important is country-of-origin for organic food consumers? A review of the literature and suggestions for future research. British Food Journal, 119(3), 542-557.

Wee, C., Ismail, K., \& Ishak, N. (2014). Consumers perception, purchase intention and actual purchase behavior of organic food products. Review of Integrative Business \& Economics Research, $3(2), 378-397$.

Zamberlan, L., Büttenbender, P., Sparemberger, A., \& Damer, I. (2010). O comportamento do consumidor de produtos orgânicos e seus impactos nas estratégias de marketing. In Sparemberger, A., Büttenbender, P., \& Zamberlan, L. (Org.). Princípios de agronegócios: conceitos e estudos de caso, 127-151. Íjuí: Unijuí. 


\begin{tabular}{|c|c|c|c|}
\hline Variáveis & $\mathbf{n}$ & Média & $\begin{array}{l}\text { Desv. } \\
\text { Pad. }\end{array}$ \\
\hline Qualidade do produto (Guimarães et al., 2013) & 219 & 4,36 & $\mathbf{0 , 8 6}$ \\
\hline Qual3. Alimentos orgânicos são mais saudáveis que os convencionais. & 219 & 4,50 & 0,92 \\
\hline Qual1. Alimentos orgânicos têm um melhor sabor/aroma que os convencionais. & 218 & 4,28 & 1,03 \\
\hline Qual4. Alimentos orgânicos são melhores que os convencionais. & 216 & 4,30 & 0,98 \\
\hline Qual2. Alimentos orgânicos têm uma melhor aparência que os convencionais. * & 217 & 3,15 & 1,21 \\
\hline Consciência Ecológica (Grohmann et al., 2012) & 219 & 4,14 & $\mathbf{0 , 8 4}$ \\
\hline Cons4. A produção orgânica auxilia o pequeno produtor. & 216 & 4,38 & 0,96 \\
\hline Cons2. Alimentos orgânicos não prejudicam o meio ambiente em sua produção. & 215 & 4,05 & 1,09 \\
\hline $\begin{array}{l}\text { Cons3. Quando eu tenho que escolher entre dois produtos iguais, eu sempre escolho o } \\
\text { que é menos prejudicial às pessoas e ao meio-ambiente. }\end{array}$ & 218 & 4,02 & 1,14 \\
\hline Cons1. Já troquei ou deixei de usar produtos por razões ecológicas. * & 217 & 3,57 & 1,42 \\
\hline Conhecimento (Hoppe et al., 2012) & 219 & 3,35 & 1,04 \\
\hline Conh4. Conheço os beneficios da produção orgânica. * & 219 & 4,06 & 1,07 \\
\hline $\begin{array}{l}\text { Conh3. Costumo ler o rótulo dos produtos, quando disponíveis, para ver se são } \\
\text { orgânicos. }\end{array}$ & 218 & 3,54 & 1,40 \\
\hline Conh1. Comparado à maioria das pessoas, sei muito sobre alimentos orgânicos. & 219 & 3,34 & 1,24 \\
\hline Conh2. Com relação a alimentos orgânicos, tenho muito conhecimento. & 217 & 3,18 & 1,13 \\
\hline Disponibilidade (Hoppe et al., 2012) & 219 & 3,13 & $\mathbf{1 , 0 0}$ \\
\hline $\begin{array}{l}\text { Disp4. Considero que há uma maior variedade de alimentos orgânicos do que alguns } \\
\text { anos atrás.* }\end{array}$ & 218 & 4,20 & 0,96 \\
\hline Disp2. Existem pontos de vendas de alimentos orgânicos de fácil acesso. & 218 & 3,39 & 1,25 \\
\hline $\begin{array}{l}\text { Disp3. Se eu quisesse, eu poderia facilmente comprar alimentos orgânicos ao invés } \\
\text { dos convencionais. }\end{array}$ & 219 & 3,04 & 1,34 \\
\hline $\begin{array}{l}\text { Disp1. Alimentos orgânicos estão geralmente disponíveis nos locais onde usualmente } \\
\text { compro alimentos. }\end{array}$ & 219 & 2,95 & 1,37 \\
\hline Preço (Perosa et al., 2009) & 219 & 3,28 & 1,03 \\
\hline Prec4. Alimentos orgânicos possuem um preço aceitável. & 218 & 3,39 & 1,14 \\
\hline $\begin{array}{l}\text { Prec1. Alimentos orgânicos tem boa relação custo-benefício (ambiental, econômico, } \\
\text { etc). }\end{array}$ & 218 & 3,72 & 1,23 \\
\hline Prec2. Alimentos orgânicos possuem preço justo. & 219 & 3,26 & 1,24 \\
\hline Prec3. Alimentos orgânicos possuem preço razoável. & 219 & 3,20 & 1,13 \\
\hline Atitude (Hoppe et al., 2012) & 219 & 4,09 & 1,09 \\
\hline $\begin{array}{l}\text { At3. Comprar alimentos orgânicos ao invés dos convencionais me faria sentir como } \\
\text { se estivesse contribuindo para algo melhor. }\end{array}$ & 218 & 4,14 & 1,10 \\
\hline $\begin{array}{l}\text { At4. Comprar alimentos orgânicos ao invés dos convencionais faria eu me sentir } \\
\text { satisfeito. }\end{array}$ & 218 & 4,11 & 1,11 \\
\hline $\begin{array}{l}\text { At2. Comprar alimentos orgânicos ao invés dos convencionais faria eu sentir que } \\
\text { estou fazendo algo correto. }\end{array}$ & 219 & 4,04 & 1,18 \\
\hline $\begin{array}{l}\text { At1. Comprar alimentos orgânicos ao invés dos convencionais faria eu me sentir uma } \\
\text { pessoa melhor. }{ }^{*}\end{array}$ & 219 & 3,79 & 1,27 \\
\hline
\end{tabular}

* Itens excluídos após os procedimentos de validação. 